\title{
Variabilidade espacial de propriedades físico-hídricas do solo, da produtividade e da qualidade de grãos de trigo em Argissolo Franco Arenoso sob plantio direto ${ }^{1}$
}

\author{
Spatial variability of soil physico-hydrical properties and wheat yield and quality in a sandy loam \\ hapludalf under no-tillage
}

\section{Silvano Luiz de Abreu ${ }^{2}$ José Miguel Reichert ${ }^{3}$ Vanderlei Rodrigues da Silva ${ }^{4}$ Dalvan José Reinert ${ }^{3}$ Elena Blume ${ }^{5}$}

\section{RESUMO}

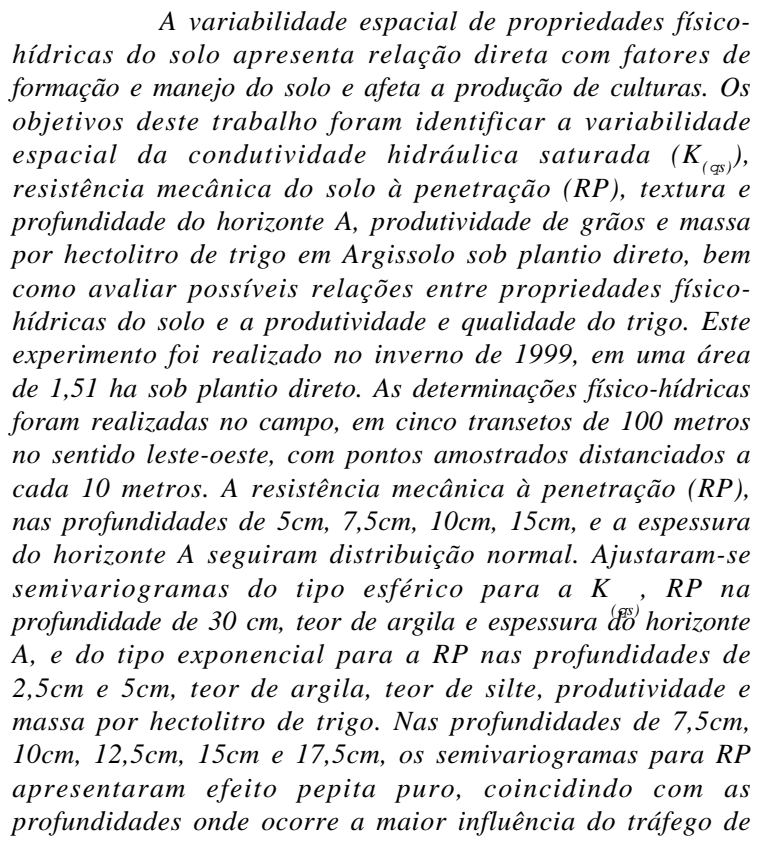

máquinas e dos sistemas de manejo do solo anteriores. A condutividade hidráulica saturada e a resistência mecânica à penetração não se correlacionaram com a produtividade de trigo. Para a avaliação da variabilidade espacial da resistência mecânica à penetração, a distância entre os pontos amostrados deverá ser inferior a 10 metros, principalmente nas profundidades maiores que $5 \mathrm{~cm}$.

Palavras-chave: geoestatística, resistência mecânica à penetração, condutividade hidráulica saturada.

\section{ABSTRACT}

Spatial varibility of soil physico-hydrical properties is related to soil genesis and management, and affect crop production. The objectives of this study were to identify and to relate spatial variability of saturated hydraulic condutivity $(K)$, soil mechanical resistance $(R)$, soil texture, and A horizont depth, of a Typic Hapludalf under no-till, with wheat yield and hectoliter weight. This experiment was conducted in the winter of 1999, on a 1.51 ha no-till wheat field. Soil and plant characterization was done on five transects with $100 \mathrm{~m}$ in the East-West direction, with sampling points $10 \mathrm{~m}$ apart. Values of $R$ for the depths of $5 \mathrm{~cm}, 7.5 \mathrm{~cm}, 10 \mathrm{~cm}$ and $15 \mathrm{~cm}$ and $A$ horizont depth showed normal distribution. Spherical model semivariograms were adjusted for $K_{(\mathrm{as})}, R$ in the $30 \mathrm{~cm}$ depth,

${ }^{1}$ Parte da dissertação de Mestrado do primeiro autor, apresentada no Programa de Pós-graduação em Agronomia, Universidade Federal de Santa Maria (UFSM).

${ }^{2}$ Engenheiro Agrônomo, Mestre, Professor Pesquisador do Curso de Agronomia ILES-ULBRA, Ji-paraná, RO. E-mail: silvano @inf.ulbrajp.com.br

${ }^{3}$ Engenheiro Agrônomo, PhD, Professor Titular do Departamento de Solos, Centro de Ciências Rurais (CCR), UFSM, 97105-900 Santa Maria, RS. Bolsista CNPq. E-mails: reichert@ccr.ufsm.br, dalvan@ccr.ufsm.br

${ }^{4}$ Engenheiro Agrônomo, Mestre, doutorando do Programa de Pós-graduação em Agronomia, UFSM. Bolsista CAPES. silvavr@bol.com.br. Autor para correspondência.

${ }^{5}$ Engenheiro Agrônomo, PhD, Professor Adjunto do Departamento de Defesa Fitossanitária, CCR, UFSM, Santa Maria, RS. Email :eblume@ccr.ufsm.br 
clay content, and A horizont depth. Exponential model for $R$ in the $2.5 \mathrm{~cm}$ and $5 \mathrm{~cm}$ depths, clay and silt content, and wheat yield and hectoliter weight. For the $7.5 \mathrm{~cm}, 10 \mathrm{~cm}, 12.5 \mathrm{~cm}$, $15 \mathrm{~cm}$ and $17.5 \mathrm{~cm}$ depths, $R$ semivariograms showed pure nugget effect; in these depths the machinery traffic and previous soil management effects are prominent. Wheat yield and hectoliter weight did not correlate with $K_{(\mathrm{cs})}$ and $R$. When evaluating the spatial variability in $R$ for soil layers deeper than $5 \mathrm{~cm}$, the distance between samples points should be smaller than $10 \mathrm{~m}$.

Key words: geostatistics, soil mechanical resistance, saturated hydraulic conductivity.

\section{INTRODUÇÃO}

O solo, por ser um corpo tridimensional formado pela ação de vários fatores e processos, apresenta uma variação de suas características ao longo da paisagem. A subdivisão de grandes extensões de solo em pequenas parcelas experimentais é uma tentativa de reduzir a variabilidade intrínseca do solo. Contudo, para o estudo de áreas maiores onde há um gradiente diferencial no solo, foi necessária a criação de técnicas amostrais e de análise de dados que levassem em consideração a variação espacial (geoestatística), que muitas vezes está associada à sua formação(WEBSTER, 1985; GOOVAERTS, 1994). Essas técnicas também são aplicadas quando o manejo do solo altera a variabilidade espacial de propriedades do solo e da planta.

A geoestatística pode ser definida como uma coleção de técnicas para a solução de problemas de estimativa envolvendo variáveis espaciais (ASCE, 1990) e tem sua base conceitual na interpretação espacial de uma variável regionalizada com realização particular de uma função aleatória. A geoestatística também permite a interpretação dos resultados obtidos do estudo com base na estrutura da sua variabilidade natural, considerando a existência de dependência espacial dentro do espaço da amostragem. O semivariograma, que é o ajuste teórico dos dados analisados a modelos matemáticos, é um dos aspectos mais importantes da aplicação da teoria das variáveis regionalizadas.

Conhecido o semivariograma da variável em estudo e havendo dependência espacial, pode-se interpolar valores em qualquer posição no campo de estudo, pelo método da krigeagem, baseando-se na função da dependência espacial (HOEKSEMA et al., 1989; WACKERNAGEL, 1993; VIEIRA, 1995). Krigeagem é um método para estimar, num contexto espacial, o valor de uma variável de interesse em um local onde não foi determinado, usando, para isso, dados obtidos em pontos adjacentes (WACKERNAGEL, 1993).
A variabilidade espacial de características hidráulicas do solo tem sido estudada por vários autores (SHARMA et al, 1980; LIBARDI et al., 1996; USOWISCZ et al., 1996; BOSCH \& WEST, 1998; HOOSBEEK, 1998; DJURHUUS et al., 1999; GONÇALVES et al., 1999). Em sua maioria, os autores encontraram relação entre as características estudadas e a paisagem. BOSCH \& WEST (1998) encontraram grande variabilidade na condutividade hidráulica saturada para dois solos arenosos, concluindo que houve maior correlação nos horizontes mais profundos e que a krigeagem foi uma ferramenta eficiente em estimar a $\mathrm{K}_{(\mathrm{g})}$ para locais não amostrados. Da mesma forma, REHFELDT et al. (1992) encontraram elevada variabilidade na condutividade hidráulica saturada em solo de várzea, cuja variabilidade foi atribuída a fatores de heterogeneidade do solo e, principalmente, à origem deposicional do solo a partir de sedimentos aluviais distintos ao longo da paisagem.

Em determinações de variabilidade espacial da taxa de infiltração de água no solo em uma bacia hidrográfica, SHARMA et al. (1980) encontraram relação entre a taxa de infiltração, sortividade e a posição do solo na paisagem. GONÇALVES et al. (1999) comentam que o uso da geoestatística se mostrou adequado ao estudo da distribuição espacial de valores de armazenamento de água no solo, embora a krigeagem sempre promova uma suavização dos valores em relação aos dados experimentais. LIBARDI et al. (1996) indicam que o conhecimento da variabilidade espacial da umidade gravimétrica do solo, antes e durante o ciclo da cultura, poderá ser indicativo da variabilidade esperada quando da determinação de propriedades dependentes do teor de água.

Em um estudo sobre a variabilidade espacial da espessura do horizonte A e da estatura e da produtividade de milho em Argissolo VermelhoAmarelo Distrófico, ALBUQUERQUE et al. (1994) verificaram dependência espacial da espessura do horizonte A e a estatura das plantas de milho. Também verificaram que as plantas foram mais altas e mais produtivas onde o horizonte A foi mais espesso. ALBUQUERQUE et al. (1996) encontraram relação direta entre a espessura do horizonte A e a produtividade de grãos de milho. A produtividade de milho foi reduzida em 42,9 $\mathrm{kg} \mathrm{ha}^{-1}$ para cada centímetro a menos na espessura do horizonte A.

Para condições de solo seco e de solo úmido, distintos comportamentos da variabilidade espacial da resistência mecânica do solo à penetração (RP) foram observados por UTSET \& CID (2001). Na condição de solo seco, obtiveram coeficiente de variação $(\mathrm{CV})$ de $25 \%$ e ajustaram semivariograma do tipo esférico, enquanto que, quando úmido, o $\mathrm{CV}$ foi de $80 \%$ e os dados apresentaram efeito pepita puro. 
Além da paisagem, o manejo influencia fortemente a variabilidade de propriedades físicas, tais como a porosidade e a $\mathrm{K}_{(\mathrm{\varphi})}$ (SLAY et al., 1986). SOUZA et al. (1997) relatam o efeito da variabilidade espacial de outras propriedades físicas (umidade gravimétrica do solo e teor de argila) e químicas (fósforo disponível e potássio) do solo na cultura da laranjeira.

Assim, percebe-se que as propriedades físico-hídricas têm grande variabilidade espaçotemporal e afetam o rendimento das culturas.

Os objetivos desse trabalho foram (i) avaliar a variabilidade espacial de propriedades físicas do solo e da produtividade do trigo, em um Argissolo, e (ii) analisar a influência de propriedades físicas do solo na produtividade e massa por hectolitro de trigo.

\section{MATERIALEMÉTODOS}

O experimento foi realizado na área experimental do Departamento de Solos, no campus da Universidade Federal de Santa Maria, Santa Maria-RS. O clima é classificado como "Cfa" de Köppen, tendo clima subtropical úmido sem estiagem.

O solo é classificado como Argissolo Vermelho-Amarelo Distrófico arênico (EMBRAPA, 1999), apresentando textura franco arenosa, no horizonte $\mathrm{A}$, e franco argilosa, no horizonte $\mathrm{B}_{\mathrm{t}}$. É originário de arenitos e siltitos, da formação Santa Maria, não apresenta pedregosidade nem rochosidade, é bem drenado e ocorre em relevo suave ondulado.

A área experimental foi cultivada em sistema convencional, com aração e gradagens durante vários anos, permaneceu em pousio por alguns anos e, nos últimos dez anos, foram instaladas lavouras de milho e soja em plantio direto, como culturas de verão, e aveia, ervilhaca e tremoço, no inverno.

O experimento foi realizado em uma área de 1,51ha, tendo $63 \mathrm{~m}$ de largura e $240 \mathrm{~m}$ de comprimento, relevo ondulado e declividade média de 7\%. Nessa mesma área, ALBUQUERQUE et al. (1996) avaliaram a variabilidade espacial de propriedades como textura do solo, profundidade do horizonte A e produtividade do milho. A área é composta por um sistema de cinco terraços de base larga distribuídos ao longo da pendente, conforme mapa apresentado em ALBUQUERQUE et al. (1996).

No verão agrícola de 1998 e 1999, na área experimental foram semeadas diversas espécies de leguminosas de verão, tais como guandu-anão, feijãode-porco e crotalarias espectabilis e juncea. Em maio de 1999, as plantas de cobertura foram roladas com rolo-faca e o trigo foi semeado diretamente sobre os resíduos culturais, sem adubação de base e sem cobertura nitrogenada, sendo que o trigo se desenvolveu apenas com o residual das plantas de cobertura de solo. Na condução da lavoura de trigo, não se utilizou nenhum herbicida, para controle de plantas espontâneas, nem pesticida, para controle de pragas e doenças.

As determinações de condutividade hidráulica saturada no campo $\left(\mathrm{K}_{(\mathrm{qs})}\right)$, resistência mecânica do solo à penetração (RP) e produtividade $\mathrm{e}$ massa por hectolitro de trigo foram realizadas nos cinco terraços, em 110 pontos distanciados a cada $10 \mathrm{~m}$ no sentido leste-oeste.

A $\mathrm{K}_{(\mathrm{gs})}$ foi determinada na profundidade de $15 \mathrm{~cm}$ em todos os pontos, no período de 27 a 29/11/ 1999, utilizando permeâmetro de Guelph (ELRICK et al., 1987). A RP foi determinada com um penetrógrafo de molas (SOILCONTROL ${ }^{\hat{a}}$-PENETROGRAPHER SC60), cone com ângulo de $30^{\circ}$ e diâmetro da base do cone de $12,83 \mathrm{~mm}$. Conjuntamente com a RP, foi determinada a umidade gravimétrica do solo, coletandose amostras em várias profundidades para cada ponto.

Os teores de argila, silte e areia na profundidade de $0 \mathrm{a} 20 \mathrm{~cm}$, determinados pelo método da pipeta, encontram-se em ALBUQUERQUE et al. (1996). Em cada coordenada da malha, coletaram-se cinco subamostras a cada $20 \mathrm{~cm}$ com trado calador em sentido transversal à linha de plantio. A determinação da profundidade do horizonte A, também realizada por ALBUQUERQUE et al. (1996) em cada coordenada da malha pelo método de sondagem com trado holandês.

A produtividade e qualidade (massa por hectolitro) de trigo foram determinadas na maturação do trigo em uma área de $2 \mathrm{~m}^{2}$ para cada ponto amostrado, nas mesmas coordenadas onde foram determinadas as propriedades físicas. As amostras de trigo foram colhidas nos dias 23 e 27/11/1999 e trilhadas mecanicamente e a umidade dos grãos foi corrigida para $13 \%$. Como a massa de grãos de uma coordenada foi insuficiente para a determinação de massa por hectolitro, juntaram-se grãos de duas coordenadas consecutivas para a determinação dessa propriedade. Assim, a distância dos pontos para as determinações de qualidade do trigo foi ampliada para $20 \mathrm{~m}$.

A variabilidade espacial desses parâmetros de solo e de planta foi analisada pelo software Geostatistics for the Environmental Sciences (Version 5.1) (GAMMA DESIGN SOFTWARE, 1998). Os semivariogramas foram ajustados conforme maior valor do coeficiente de correlação obtido pelo método de validação cruzada, o qual consiste em retirar, individualmente, cada ponto medido da área estudada e o seu valor é estimada via krigeagem como se ele não existisse.

Ciência Rural, v. 33, n. 2, mar-abr, 2003. 
$\mathrm{Na}$ análise da estatística descritiva, valores de média, moda, mediana, coeficiente de variação, curtose, assimetria e teste $\mathrm{W}$ foram quantificados pelo pacote estatístico SAS (Statistical Analysis System, 1985).

\section{RESULTADOSEDISCUSSÃO}

A resistência mecânica à penetração $(\mathrm{RP})$, nas profundidades de $5 \mathrm{~cm}, 7,5 \mathrm{~cm}, 10 \mathrm{~cm}$ e $15 \mathrm{~cm}$, e a espessura do horizonte $\mathrm{A}$ apresentaram distribuição normal (Tabela 1), conforme análise do teste $\mathrm{W}$ (Kolmogorow) e análise visual dos valores de média, mediana e moda. UTSET \& CID (2001) também verificaram em um latossolo, que a RP apresentou distribuição normal. OLIVEIRA et al. (1999) observaram distribuição normal somente para uma de onze propriedades químicas do solo estudadas e, segundo SOUZA et al. (1997), essas diferenças no tipo de distribuição são atribuídas ao nível e à forma da variabilidade do solo. De acordo com WARRICK \& NIELSEN (1980), geralmente as propriedades mecânicas do solo apresentam distribuição normal, enquanto propriedades hidráulicas apresentam distribuição não normal.

Usando os valores propostos por WARRICK \& NIELSEN (1980), os coeficientes de variação foram, em geral, considerados médios para as variáveis. Apenas as variáveis $K_{(\mathrm{qs})}$ e RP na profundidade de $30 \mathrm{~cm}$ apresentaram $\mathrm{CV}$ alto. Os valores de areia e silte apresentaram um coeficiente de variação

Tabela 1 - Valores de média, mediana (Med), moda (Mo), coeficiente de variação (CV em \%), assimetria (Ass), curtose (Curt) e teste W para todas as propriedades avaliadas em solo sob sistema de manejo conservacionista.

\begin{tabular}{|c|c|c|c|c|c|c|c|}
\hline Variável & Média & Med & Mo & $\mathrm{CV}$ & Ass & Curt & $\mathrm{W}$ \\
\hline $\mathrm{K}_{\left(\theta_{\mathrm{s})}\right.}\left(\mathrm{cm} \mathrm{h}^{-1}\right)$ & 10,63 & 9,53 & 3,06 & 62,7 & 0,58 & $-0,22$ & $0,946^{\mathrm{ns} 1}$ \\
\hline $\mathrm{RP} 2,5 \mathrm{~cm}(\mathrm{MPa})$ & 0,95 & 0,83 & 0,70 & 49,6 & 0,67 & $-0,05$ & $0,930^{\mathrm{ns}}$ \\
\hline RP 5 cm (Mpa) & 1,73 & 1,73 & 1,95 & 38,9 & $-0,16$ & 0,37 & $0,978 * 2$ \\
\hline $\mathrm{RP} 7,5 \mathrm{~cm}(\mathrm{MPa})$ & 2,23 & 2,25 & 2,10 & 27,5 & 0,25 & $-0,23$ & $0,967 *$ \\
\hline $\mathrm{RP} 10 \mathrm{~cm}(\mathrm{MPa})$ & 2,38 & 2,40 & 1,95 & 30,7 & 0,12 & $-0,57$ & $0,973 *$ \\
\hline $\mathrm{RP} 12,5 \mathrm{~cm}(\mathrm{MPa})$ & 2,43 & 2,40 & 2,10 & 32,7 & 0,25 & 0,73 & $0,951^{\mathrm{ns}}$ \\
\hline $\mathrm{RP} 15 \mathrm{~cm}(\mathrm{MPa})$ & 2,47 & 2,53 & 1,95 & 33,4 & 0,34 & 0,07 & $0,969^{*}$ \\
\hline $\mathrm{RP} 17,5 \mathrm{~cm}(\mathrm{MPa})$ & 2,52 & 2,40 & 2,10 & 43,4 & 2,92 & 18,3 & $0,829^{\mathrm{ns}}$ \\
\hline $\mathrm{RP} 30 \mathrm{~cm}(\mathrm{MPa})$ & 2,32 & 1,80 & 1,10 & 82,9 & 2,77 & 8,76 & $0,695^{\mathrm{ns}}$ \\
\hline Areia $\left(\mathrm{kg} \mathrm{kg}^{-1}\right)$ & 628 & 634 & 627 & 6,96 & $-1,84$ & 4,59 & $0,850^{\mathrm{ns}}$ \\
\hline Silte $\left(\mathrm{kg} \mathrm{kg}^{-1}\right)$ & 275 & 273 & 245 & 11,2 & 1,29 & 4,13 & $0,937^{\mathrm{ns}}$ \\
\hline Argila $\left(\mathrm{kg} \mathrm{kg}^{-1}\right)$ & 97,5 & 87,4 & 81,5 & 27,9 & 1,34 & 0,64 & $0,798^{\text {ns }}$ \\
\hline $\begin{array}{l}\text { Espessura do } \\
\text { horizonte A (m) }\end{array}$ & 0,73 & 0,75 & 0,90 & 28,5 & $-0,15$ & $-0,62$ & $0,967 *$ \\
\hline $\begin{array}{l}\text { Produtividade de } \\
\text { trigo }\left(\mathrm{Mg} \mathrm{ha}^{-1}\right)\end{array}$ & 0,79 & 0,78 & 0,93 & 25,5 & 0,42 & 0,56 & $0,962^{\mathrm{ns}}$ \\
\hline $\begin{array}{l}\text { Massa por } \\
\text { hectolitro }\left(\mathrm{kg} \mathrm{hl}^{-1}\right)\end{array}$ & 72,4 & 73,0 & 65,8 & 2,75 & $-1,46$ & 2,38 & $0,867^{\mathrm{ns}}$ \\
\hline
\end{tabular}

${ }^{1}$ Variável segue distribuição não normal.

${ }^{2}$ Variável segue distribuição normal.
(CV) baixo, à semelhança do observado por CARVALHO et al. (1998).

O uso do semivariograma para a análise geoestatística permitiu verificar a presença ou não de dependência espacial nas propriedades analisadas. Se o semivariograma, ao invés de ser crescente e dependente da distância, for constante e igual ao patamar para qualquer distância, tem-se ausência total de dependência espacial na escala estudada, também chamado de efeito pepita puro (VIEIRA, 2000). Esse fenômeno foi verificado para a variável RP nas profundidades de $10 \mathrm{~cm}$ (Figura 1 ) e de $7,5 \mathrm{~cm}, 12,5 \mathrm{~cm}$, $15 \mathrm{~cm}$ e $17,5 \mathrm{~cm}$ (dados não apresentados). Para essas variáveis, a variabilidade do solo é muito grande, necessitando de um esquema de amostragem em pontos mais próximos que o utilizado neste estudo, ou de um número maior de repetições, pois a RP apresenta grande variabilidade entre as repetições.

Quando o semivariograma é crescente e dependente da distância, deve-se ajustar um modelo que melhor ilustra o comportamento da variabilidade da variável em função da distância. O semivariograma é dividido em três regiões: i) a interceptação do semivariograma com o eixo das ordenadas chamado de efeito pepita $\left(\mathrm{C}_{\mathrm{o}}\right)$ indica a variação ao acaso ou erros na obtenção dos dados; ii) o patamar $\left(\mathrm{C}_{\mathrm{o}}+\mathrm{C}_{1}\right)$, que é a estabilização dos valores do semivariograma, aproximadamente igual à variância dos dados e; iii) o alcance (a), que é a distância onde o semivariograma alcança o patamar. $\mathrm{O}$ alcance indica a distância limite entre pontos correlacionados entre si. Pontos distanciados maiores que o alcance são independentes e para sua análise podese utilizar a estatística clássica (VIEIRA, 2000).

Ajustou-se o modelo exponencial para os semivariogramas das variáveis $\mathrm{RP}$ a $2,5 \mathrm{~cm}, 5 \mathrm{~cm}$, areia, silte e produtividade e massa por hectolitro de trigo. Para as variáveis $\mathrm{K}_{(\mathrm{os})}$, teor de argila, RP na profundidade de $30 \mathrm{~cm}$ e espessura do horizonte A, ajustaram-se semivariogramas do tipo esférico.

Para analisar o grau de dependência espacial das variáveis em estudo, realizou-se uma modificação da classificação de CAMBARDELLA et al. (1994), subtraindo uma unidade do resultado da divisão entre $\mathrm{C}_{\mathrm{o}}$ e $\mathrm{C}_{\mathrm{o}}+\mathrm{C}_{1}$. Nesse caso, são considerados de dependência espacial forte os semivariogramas que têm um efeito pepita 


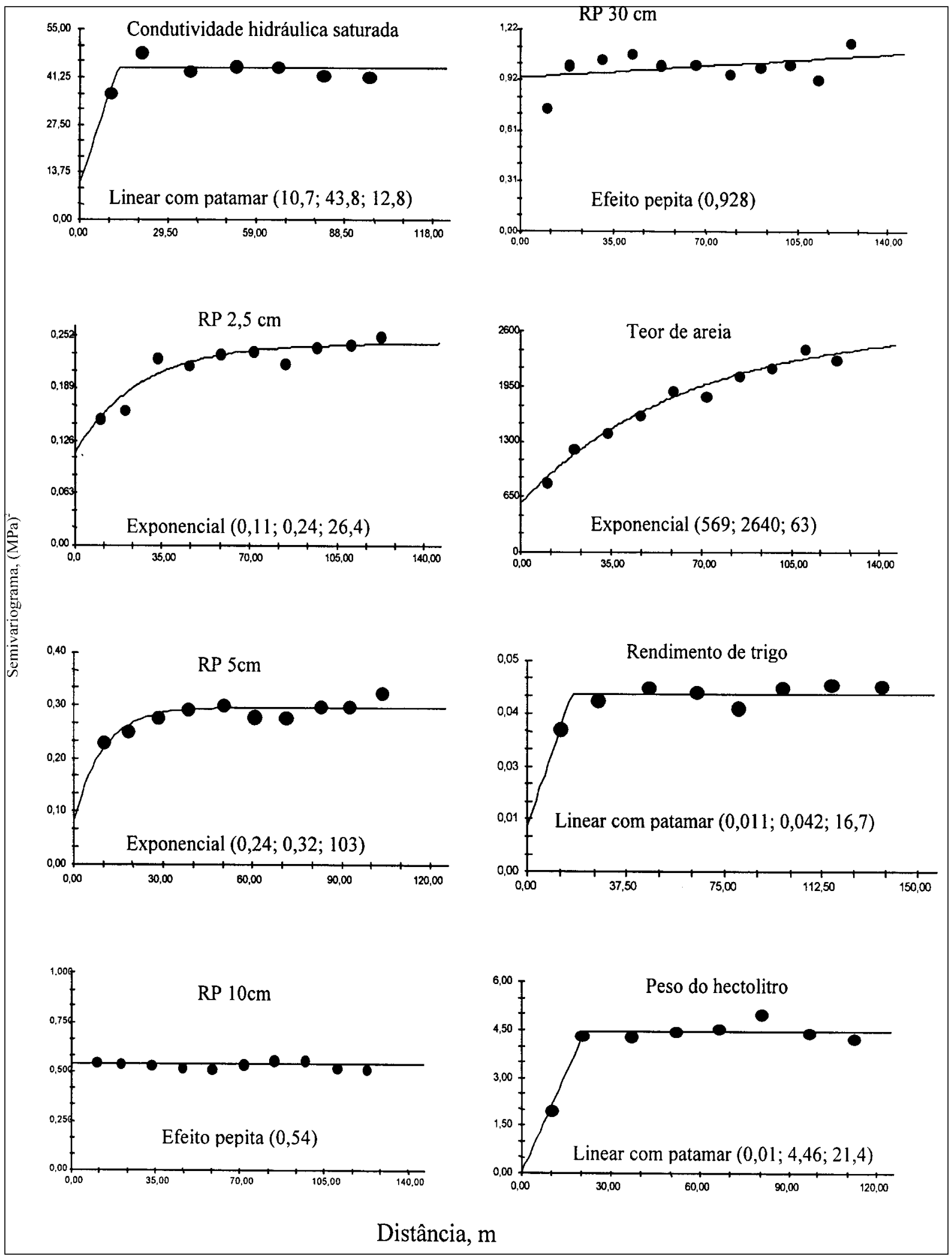

Figura 1 - Semivariogramas ajustados para as as propriedades de solo e planta. (Entre parênteses, encontram-se os valores do efeito pepita, do patamar e do alcance do semivariograma ajustado aos dados de semivariância).

Ciência Rural, v. 33, n. 2, mar-abr, 2003. 
> 0,75 do patamar, de dependência espacial moderada quando o efeito pepita está entre 0,74 e 0,26 e de dependência fraca quando o efeito pepita é $<0,25$. Os semivariogramas ajustados para RP nas profundidades de $2,5 \mathrm{~cm}$ e $5 \mathrm{~cm}$ e silte apresentaram moderada dependência espacial, em torno de 0,5. Para areia, $\mathrm{K}_{\mathrm{(qs}}$, RP na profundidade de $30 \mathrm{~cm}$ produtividade e massa por hectolitro de trigo, os semivariogramas foram classificados de dependência forte (Tabela 2). De uma maneira geral, propriedades do solo apresentam moderada dependência espacial.

Analisando a variabilidade

espacial da RP nas diferentes profundidades, pode-se inferir que a compactação do solo causada pelo efeito acumulativo das pressões nos mais de 10 anos de plantio direto tenha diminuído sensivelmente a variabilidade espacial na subsuperfície, predominando a variabilidade aleatória. Em sistema de plantio direto, essa camada $(7,5 \mathrm{a} 15 \mathrm{~cm})$ é a que sofre maior compactação (HAKANSSON et al., 1988; SILVA et al., 2000). CADIMA et al. (1980) também observaram maior variabilidade com o aumento na profundidade no perfil do solo.

ALBUQUERQUE et al. (1996) ajustaram modelos esféricos para o teor de argila e espessura do horizonte A, com alcance de 39 metros para o teor de argila e 80 metros para a espessura do horizonte A. O alcance da dependência espacial foi de $26,4 \mathrm{~m}$ para RP a $2,5 \mathrm{~cm}, 278,4 \mathrm{~m}$ para $\mathrm{RPa} 5 \mathrm{~cm}$, e de $18,4 \mathrm{~m}$ para $R P a 30 \mathrm{~cm}$ de profundidade. Ao analisarmos todas as profundidades, inclusive aquelas com efeito pepita puro, visualizamos que a RP nesta área é bastante variável, principalmente pelas diferenças na espessura do horizonte A, na quantidade de matéria orgânica e na granulometria da área. Para as variáveis granulométricas do solo, o alcance ficou entre 45 e $67 \mathrm{~m}$. Os menores valores de alcance foram para as variáveis $\mathrm{K}_{(\mathrm{qs})}$ e produtividade e a massa por hectolitro de trigo, entre 15 e 10,9m.

Analisando somente o terceiro e quinto terraços da área do presente estudo, ALBUQUERQUE et al. (1996) encontraram dependência espacial da produtividade do milho, o qual estava associado à profundidade do horizonte A; para cada cm de solo erodido houve uma relação de decréscimo de $42,9 \mathrm{~kg}$ $\mathrm{ha}^{-1}$ de milho. No presente estudo, entretanto, o coeficiente de correlação entre produtividade do trigo e espessura do horizonte A foi significativo somente a $10 \%$ de probabilidade, correspondendo somente $20 \%$ da variação. A redução na produtividade de trigo foi de aproximadamente $2 \mathrm{~kg} \mathrm{ha}^{-1}$ para cada centímetro a menos na espessura do horizonte A.

No segundo terraço, a massa por hectolitro do trigo correlacionou-se positivamente com a percentagem de areia $(\mathrm{r}=0,74 * * *)$ e profundidade do horizonte $\mathrm{A}\left(\mathrm{r}=0,43^{*}\right)$ e negativamente com os teores de argila $(\mathrm{r}=-0,64 * *)$ e silte $\left(\mathrm{r}=-0,68^{* * *}\right)$. Esses resultados e aqueles obtidos por ALBUQUERQUE et al. (1996) indicam a necessidade de manejo variável da adubação e da irrigação, para otimizar o uso de nutrientes e da água.

O trigo desenvolveu-se apenas com o residual dos nutrientes disponibilizados pelas plantas de cobertura de solo, sem aplicação de fungicidas, que pode explicar sua baixa produtividade em ano com condições climáticas adversas, embora a massa por hectolitro ser considerada boa para as condições edafoclimáticas do local do experimento.

\section{CONCLUSÕES}

A resistência mecânica do solo à penetração (RP) apresentou ausência total de dependência espacial na camada sob maior estado de compactação, que ocorria de 7,5 a 17,5cm de profundidade. Na camada superficial de $2,5 \mathrm{~cm}$ a $5 \mathrm{~cm}$ e na profundidade de $30 \mathrm{~cm}$, os dados de resistência mecânica à penetração apresentaram dependência espacial, ajustando-se modelo exponencial com alcance de $26,4 \mathrm{~m}$ e $103 \mathrm{~m}$, respectivamente para a profundidade de $2,5 \mathrm{~cm}$ e $5 \mathrm{~cm}$, e modelo esférico com alcance em torno de $18 \mathrm{~m}$ na 
profundidade de $30 \mathrm{~cm}$.

Para a avaliação da variabilidade espacial da resistência mecânica à penetração, principalmente nas profundidades entre $5 \mathrm{~cm} 17,5 \mathrm{~cm}$, a distância entre os pontos amostrados deverá ser inferior a 10 metros.

A condutividade hidráulica saturada e a resistência mecânica à penetração não correlacionaram com a produtividade de trigo, em ano com clima adverso à cultura, havendo uma pequena redução nessa produtividade (aproximadamente $2 \mathrm{~kg} \mathrm{ha}^{-1}$ ) para cada centímetro de decréscimo na espessura do horizonte A.

\section{REFERÊNCIASBIBLIOGRÁFICAS}

ALBUQUERQUE, J.A. et al. Variabilidade espacial em parcelas experimentais de perda de água e solo por chuva natural. Ciência Rural, Santa Maria, v.24, n.2, p.269-273, 1994.

ALBUQUERQUE, J.A.; REINERT, D.J.; FIORIN, J.E. Variabilidade de solo e planta em podzólico vermelho-amarelo. Revista Brasileira de Ciência do Solo, Viçosa, v.20, p.151157, 1996.

ASCE. Review of geostatistics in geohydrology. I: Basics concepts. Journal of Hydraulic Engineering, New York, v. 116, n.5, p.612-632, 1990.

BOSCH, D.D.; WEST, L.T. Hydraulic conductivity variability for two sandy soils. Soil Science Society of America Journal, Madison, v.62, p.90-98, 1998.

CADIMA, A.; LIBARDI, P.L.; REICHARDT, K. Variabilidade espacial da condutividade hidráulica em um latossolo vermelhoamarelo textura média, no campo. Revista Brasileira de Ciência do Solo, Campinas, v.4, n.2, p.63-66, 1980.

CAMBARDELLA, C.A. et al. Field scale variability of soil properties in Central Iowa soils. Soil Science Society of America Journal, Madison, v.58, p.1501-1511, 1994

CARVALHO, O.S. et al. Variabilidade espacial de algumas propriedades químicas e físicas de um solo submetido a diferentes sucessões de cultivo. Revista Brasileira de Ciência do Solo, Viçosa, v.22, n.3, p.497-503, 1998.

DJURHUUS, J. et al. Modeling mean nitrate leaching from spatially variable fields using effective hydraulic parameters. Geoderma, Amsterdan, v.87, n.4, p.261-279, 1999.

ELRICK, D.E. et al. In-situ measurements of hydraulic properties of soils using the Guelph Permeameter and the Guelph Infiltrometer, Ohio, 1987. In: INT. WORKSHOP ON LAND DRAINAGE, 3., 1987, Ohio. Anais... Ohio : Ohio State Univ, 1987. p.13-23.

EMPRESA BRASILEIRA DE PESQUISA AGROPECUÁRIAEMBRAPA. Centro Nacional de Pesquisa de Solos. Sistema brasileiro de classificação de solos. Rio de Janeiro, 1999. $412 \mathrm{p}$.

GAMMA DESIGN SOFTWARE. Geoestatistics for the environmental sciences (version 5.1 for windows). Michigan, 2000 .

GONÇALVES, A.C.A.; FOLEGATTI, M.V.; VIEIRA, S.R. Padrões de amostragem e intensidade de krigeagem em caracterização do armazenamento de água no solo, em área irrigada por pivô central. Revista Brasileira de Ciência do Solo, Viçosa, v.23, n.3, p.485-495, 1999.

GOOVAERTS, P. Study of spatial relationships between two sets of variables using multivariate geostatistics. Geoderma, Amsterdan, v.62, p.93-107, 1994.

HAKANSSON, I.; VOORHEES, W.B.; RILEY, H. Vehicle and wheel factors influencing soil compaction and crop response in different traffic regimes. Soil \& Tillage Research, Amsterdam, v.11, p.239-282, 1988.

HOEKSEMA, R.J. et al. Cokriging model for estimation of water table elevation. Water Resource Research, Washington, v.25, n.3, p.429-438, 1989.

HOOSBEEK, M.R. Incorporating scale into spatio-temporal variability: applications to soil quality and yield data. Geoderma, Amsterdan, v.85, n.2, p.113-131, 1998.

LIBARDI, P.L. et al. Variabilidade da umidade gravimétrica de um solo hidromórfico. Revista Brasileira de Ciência do Solo, Viçosa, v.20, n.1, p.1-12, 1996.

OLIVEIRA, J.J. et al. Variabilidade espacial de propriedades químicas em um solo salino-sódico. Revista Brasileira de Ciência do Solo, Viçosa, v.23, p.783-789, 1999.

REHFELDT, K.R.; BOGGS, J.M.; GELHAR, L.W. Field study of dispersion in a heterogeneous aquifer 3. Geostatistics analysis of hydraulic conductivity. Water Resource Research, Washington, v.28, n.12, p.3309-3324, 1992.

SHARMA, M.L.; GANDER, G.A.; HUNT, C.G. Spatial variability of infiltration in a watershed. Geoderma, Amsterdan, v.45, p.101-122, 1980.

SAS Institute. SAS User's guide; statistics. 5 ed. Cary, N.C., 1985. 956p.

SILVA, V.R.; REINERT, D.J.; REICHERT, J.M. Resistência mecânica do solo à penetração influenciada pelo tráfego de uma colhedora em dois sistemas de manejo do solo. Ciência Rural, Santa Maria, v.30, n.5, p.795-801, 2000.

SLAY, J.M. et al. The influence of pretreatment forest soil variability on interpretation of posttreatment results. Soil Science Society of America Journal, Madison, v.50, p.15941597,1986

SOUZA, L. da S.; COGO, N.P.; VIEIRA, S.R. Variabilidade de propriedades físicas e químicas do solo em um pomar cítrico. Revista Brasileira de Ciência do Solo, Viçosa, v. 21, n.3, p.367-372, 1997.

USOWICZ, B.; KOSSOWSKI, J.; BARANOWSKI, P. Spatial variability of soil thermal properties in cultivated fields. Geoderma, Amsterdan, v.39, n.2, p.85-100, 1996.

UTSET, A.; CID, G. Soil penetrometer resistance spatial variability in a Ferrosol at several soil moisture conditions. Soil \& Tillage Research, Amsterdam, v.61, p.193-202, 2001.

Ciência Rural, v. 33, n. 2, mar-abr, 2003. 
VIEIRA, S.R. Geoestatística em estudos de variabilidade espacial do solo. In: NOVAIS, P.F.; ALVAREZ, V.H.; SCHAEFER, C.E.G.R. Tópicos em ciência do solo. Viçosa : Sociedade Brasileira de Ciência do Solo, 2000. V.1. p.1-54.

WARRICK, A.W.; NIELSEN, D.R. Spatial variability of soil physical properties in the field. In: HILLEL, D. (ed.).
Applications of soil physics. New York : Academic, 1980.

WARRICK, A.W.; MYERS, D.E.; NIELSEN, D.R. Geostatistical methods applied to soil science. In: KLUTE, A. (ed). Methods of soil analysis. Part 1 - Physical and Mineralogical Methods. 2 ed. Madison : ASA and SSSA, 1986. p.5381.

WEBSTER, R. Quantitatives spatial analylis of soil en the field. Advances in Soil Science, New York, 1985. p.1-70. 